\title{
Modeling and Analysis of Single-Phase Induction Machines with Dynamic Phasors
}

\author{
A.M. STANKOVIC ${ }^{\dagger}$ \\ B.C. Lesieutre ${ }^{\dagger \dagger}$ \\ T. AYDIN ${ }^{\dagger}$ \\ $\dagger$ Northeastern University \\ t† Massachusetts Institute of Technology
}

\begin{abstract}
In this paper we present applications of the phasor-dynamic (or generalized averaging) modeling technique to single-phase induction machines. We are interested in a derivation of large and small-signal models, as this class of machines exemplifies electromechanical systems in which lack of symmetry in the magnetic field produced by the windings prevents the use of standard simplifying assumptions (such as the smoothness of the electromagnetic torque). In the process we derive equivalent circuits that do not require superposition-type assumptions common in the literature. The simplest of these circuits coincides with the standard one. We also present and quantify refinements that could be useful for evaluation of efficiency, and in estimation and control of single-phase induction machines.
\end{abstract}

Key Words -phasor dynamics, generalized averaging, induction machine, electromechanical systems.

\section{INTRODUCTION}

In this paper we develop dynamic models and equivalent circuits for single-phase induction motors through application of the generalized averaging technique. This approach offers several advantages, as it isolates harmonic behavior present in unbalanced machinery, and allows for more accurate calculation of efficiency. Importantly, from a modeling point of view, the derivations do not involve superposition-type assumptions that are common in textbooks on electric machinery.

The single-phase induction motor is the most widely used type of motor. It is used in most household appliances that require motors, for example in refrigerators, washers, driers,

PE-379-PWRS-0-2-1998 A paper recommended and approved by the IEEE Power Engineering Education Committee of the IEEE Power Engineering Society for publication in the IEEE Transactions on Power Systems. Manuscript submitted August 1, 1997; made available for printing March 2, 1998 fans and blowers. An interesting property of this type of motor is that the stator current alone does not create a revolving magnetic field - a sinusoidal input will produce a pulsating magnetic field along a single axis. If the machine is at standstill, this excitation will not provide any torque.

The classical "rotating field theory" states that this pulsating wave can be expressed as two magnetic fields rotating in opposite directions. Each field acts upon the rotor as it would for a balanced poly-phased induction motor. When the rotor is stationary (blocked), the forward and backward fields are equal in strength. When the rotor is turning in the forward direction, the induced currents in the rotor act to increase the strength of the forward rotating field, and to decrease the strength of backward rotating field. Balanced induction machine models for each field are combined to get an overall circuit that reflects this phenomenon. Similarly, the torque components due to the forward and backward rotating fields are added to obtain the total average torque.

An examination of textbooks on electric machinery reveals that "rotating field theory" is by far the most common approach to explaining and deriving equivalent circuits for single-phase induction motors. (For a representative sample see [1] - [8].) This well-established procedure is in common use. An alternative approach to the study of these motors, called "cross-field theory" [9], does not offer a different, or more accurate equivalent circuit, and will not be discussed further.

Here we state a fundamental objection to the traditional derivation of the equivalent circuit for the single-phase induction motor: by decomposing a quantity (magnetic field)into a sum of separate components (forward and backward rotating fields), analyzing the components separately (torque due to forward and backward rotating fields) and summing the results to achieve a total response (average torque), one is applying the principle of superposition. In system-theoretic terms, superposition can only be applied to linear systems. An induction motor is a nonlinear systern - for example, doubling the voltage and load torque inputs will not exactly double stator current and machine speed outputs, and halving the applied voltage and load torque may cause the motor to stall. 
Pedagogically, it may seem inappropriate to introduce superposition in a linear systems course, and then apply it to a nonlinear system in an electric machinery course without further justification, or error analysis. In practical engineering terms, we are interested in possible errors caused by this application of superposition.

To better understand the effect of the nonlinearities, consider the application of a fixed voltage source of fundamental frequency. This will tend to produce stator currents at fundamental frequency which in turn will produce constant and second harmonic torques on the shaft (provided the rotor is not at standstill). The effect of the higher frequency torque will be attenuated by the inertia of the machine, but will produce some second harmonic rotational speed. This speed variation will introduce additional first and third harmonic currents in the windings, and so on. The traditional derivation does not allow for careful examination of these harmonic interactions.

We propose an approach that explicitly addresses the harmonic composition of signals of interest. Our modeling approach has originated in power electronics $[10,11]$, and has been applied to flexible $\mathrm{AC}$ transmission systems [12]. The main idea, applicable to systems with (close to) periodic inputs, is to represent the system response as a Fourier series with (slowly) time-varying coefficients. The dynamical equations are then derived for the Fourier coefficients. Various forms of frequency selective analysis have deep roots in power engineering in the form of phasor-based dynamical models. The main advantage of generalized averaging is its systematic derivation of phasor dynamics. The dynamical equations for Fourier coefficients are often nonlinear, and their analytical usefulness stems from the availability of families of approximations that are based on physical insights offered by the underlying frequency decomposition.

The possibility of using time-varying Fourier coefficients in dynamical analysis of electrical machinery is mentioned in [13], but it is not pursued analytically. We illustrate the usefulness of the generalized averaging methodology in deriving large and small-signal dynamical models and equivalent circuits for a single-phase induction machine. Simulations are used to verify the accuracy of the large signal model and also serve to demonstrate the potential computational advantages offered by slowly varying dynamic phasor models. We compare the linearization of the dynamic phasor model in a steady state with the linearization of the standard representation based on Floquet theory. The equivalent circuits are dexived from the dynamic equations without assuming superposition, and are used to calculate the machine efficiency.

Our paper is organized as follows: in Section II we describe the generalized averaging method; in Section III we present the conventional model of a single-phase induction machine in a stationary reference frame; in Section IV we apply the averaging method of Section II to the model presented in Section III; simulation results are presented in Section IV, while in Section V we analyze small-signal models of of a singlephase induction machine; in Section VI we derive two equivalent circuits (the simpler of which coincides with the conventional equivalent circuit) without requiring the superposition of torques produced by forward and backward rotating fields.

\section{Time-Varying Fourier Coefficients}

The generalized averaging that we perform to obtain our models is based on the property $[10,11]$ that a waveform $x(\tau)$ can be represented on the interval $\tau \in(t-T, t]$ using a Fourier series of the form

$$
x(\tau)=\sum_{k=-\infty}^{\infty} X^{k}(t) e^{j k \omega_{b} \tau}
$$

where $\omega_{b}=2 \pi / T$ and $X^{k}(t)$ are the complex Fourier coefficients, which we shall also refer to as phasors. These Fourier coefficients are functions of time since the interval under consideration slides as a function of time. The $k$-th coefficient (or $k$-phasor) at time $t$ is determined by the following averaging operation:

$$
X^{k}(t)=\frac{1}{T} \int_{t-T}^{t} x(\tau) e^{-j k \omega_{b} \tau} d \tau=<x>_{k}(t) .
$$

The notation $\langle x\rangle_{k}(t)$ will be used to denote the averaging operation in (2). We omit the explicit dependence upon time in the rest of the paper. Our analysis provides a dynamic model for the dominant Fourier series coefficients as the window of length $T$ slides over the waveforms of interest. More specifically, we obtain a state-space model in which the coefficients in (2) are the state variables. We note that since our original variables $x(\cdot)$ are real-valued, the phasor $X^{-k}$ is the complex conjugate of $X^{k}$.

A key fact for our development is that the derivative of the $k$-th Fourier coefficient is given by the following expression:

$$
\frac{d X^{k}}{d t}=\left\langle\frac{d}{d t} x\right\rangle_{k}-j k \omega_{b} X^{k}
$$

This formula is easily verified using (1) and (2).

\section{Modeling of A Single-Phase Induction Motor USING DYNAMIC Phasors}

Assuming a sinusoidal stator voltage and shorted rotor windings, then the following model can be written (in a stationary reference frame [13]):

$$
\begin{aligned}
\sqrt{2} V \cos \left(\omega_{b} t\right)= & r_{s} i_{q s}+\frac{X_{s}}{\omega_{b}} \frac{d i_{q s}}{d t}+\frac{X_{m}}{\omega_{b}} \frac{d i_{q r}}{d t} \\
0= & r_{r} i_{q r}-\frac{\omega_{r}}{\omega_{b}} X_{r} i_{d r}+\frac{X_{r}}{\omega_{b}} \frac{d i_{q r}}{d t}+ \\
& \frac{X_{m}}{\omega_{b}} \frac{d i_{q s}}{d t}
\end{aligned}
$$




$$
\begin{aligned}
0= & r_{r} i_{d r}+\frac{\omega_{r}}{\omega_{b}}\left(X_{r} i_{q r}+X_{m} i_{q s}\right)+ \\
& \frac{X_{r}}{\omega_{b}} \frac{d i_{d r}}{d t} \\
\frac{2 H}{\omega_{b}} \frac{d \omega_{r}}{d t}= & -T_{L}-B_{f} \omega_{r}+X_{m} i_{q s} i_{d r}
\end{aligned}
$$

where $i_{q s}$ represents the stator current, $i_{d r}$ and $i_{q r}$ are the rotor winding currents referenced to the stator, and $\omega_{r}$ is the rotor velocity.

The model (4) can be written in a state-space form: if we define the state $x=\left[\begin{array}{llll}i_{q s} & i_{q r} & i_{d r} & \omega_{r}\end{array}\right]^{T}$, then the model reveals the following structure:

$$
\begin{aligned}
\dot{x_{1}} & =a_{11} x_{1}+a_{12} x_{2}+a_{13} x_{3} x_{4}+b_{1} \cos \left(\omega_{b} t\right) \\
\dot{x_{2}} & =a_{21} x_{1}+a_{22} x_{2}+a_{23} x_{3} x_{4}+b_{2} \cos \left(\omega_{b} t\right) \\
\dot{x_{3}} & =a_{31} x_{1} x_{4}+a_{32} x_{2} x_{4}+a_{33} x_{3} \\
\dot{x_{4}} & =a_{41} x_{1} x_{3}+a_{44} x_{4}+b_{4} T_{L}
\end{aligned}
$$

We can express each of the state variables in terms of a Fourier series with (slowly) time-varying coefficients. To make calculations tractable, we have to decide on the number of harmonics that should be retained for a desired model accuracy. This is often based on physical intuition; error bounds for general systems are given in [11]. To develop our phasor-dynamic model, we will assume a constant load torque $T_{L}$, keep first $( \pm 1)$ harmonic for all currents $x_{1}-x_{3}$ and $\mathrm{dc}$ component and second $( \pm 2)$ harmonic for speed $x_{4}$. This set of assumptions can be deduced, for example, from numerical simulations. From (5), in steady state our assumptions amount to neglecting third (and higher) harmonics in currents, which is often quite reasonable.

Let $X_{1}^{1}-X_{3}^{1}$ denote Fourier coefficients corresponding to first (fundamental) harmonics of states $x_{1}-x_{3}$, and let $X_{4}^{0}$ and $X_{4}^{2}$ denote the zeroth $(\mathrm{dc})$ and second Fourier coefficient of the speed $x_{4}$. Let $z^{*}$ and $\Re(z)$ denote the complex conjugate and the real part, respectively, of a quantity $z$, and recall that $X_{\ell}^{-k}=X_{\ell}^{k *}$. (Note that the subscript identifies the variable, while the superscript refers to the harmonic number.) Application of (2) and (3) to our model (5) then yields:

$$
\begin{aligned}
\dot{X}_{1}^{1}= & \left(a_{11}-j \omega_{b}\right) X_{1}^{1}+a_{12} X_{2}^{1}+a_{13} X_{3}^{1} X_{4}^{0}+ \\
& a_{13}\left(X_{3}^{1}\right)^{*} X_{4}^{2}+\frac{b_{1}}{2} \\
\dot{X_{2}^{1}=} & a_{21} X_{1}+\left(a_{22}-j \omega_{b}\right) X_{2}+a_{23} X_{3}^{1} X_{4}^{0}+ \\
& a_{23}\left(X_{3}^{1}\right)^{*} X_{4}^{2}+\frac{b_{2}}{2} \\
\dot{X_{3}^{1}=} & a_{31} X_{1}^{1} X_{4}^{0}+a_{31}\left(X_{1}^{1}\right)^{*} X_{4}^{2}+a_{32} X_{2}^{1} X_{4}^{0}+ \\
& a_{31}\left(X_{2}^{1}\right)^{*} X_{4}^{2}+\left(a_{33}-j \omega_{b}\right) X_{3}^{1} \\
\dot{X_{4}^{0}=} & 2 a_{41} \Re\left(X_{1}^{1}\left(X_{3}^{1}\right)^{*}\right)+a_{44} X_{4}^{0}+b_{4} T_{L} \\
\dot{X_{4}^{2}=} & a_{41} X_{1}^{1} X_{3}^{1}+\left(a_{44}-j 2 \omega_{b}\right) X_{4}^{2}
\end{aligned}
$$

This fifth order model involves complex quantities, and it can be replaced by a ninth order model involving real quantities (observe that $X_{4}^{0}$ is real). A simpler (but still quite accurate) fourth order (coupled) model is obtained if only zero-th (dc) component of the speed is retained (i.e., if one sets $X_{4}^{2}=0$ ).

\section{Simulation Results}

Note that both models (5) and (6) are nonlinear. One major advantage of model (6) is in simulations, as phasor quantities involved tend to vary slowly, so that large step sizes are allowed in solving the differential equations. While we have not quantified the issue in detail, we have routinely observed an order of magnitude improvement in our simulations of (6) compared to (5).

To illustrate the results obtained with models (5) and (6) we use the numerical example of a $1 / 4$ horse power machine from $[13, \mathrm{p}$. 438] (we set the moment of inertia $J$ to $0.0015 \mathrm{kgm}^{2}$ ). We consider the case in which the machine is loaded with a constant $T_{L}=0.8 \mathrm{Nm}$, and then, at $t=2 \mathrm{~s}$, the load torque steps to the value of $0.2 \mathrm{Nm}$. In all plots we compare the detailed simulations from (5) with calculations of the envelopes obtained from (6). We observe an

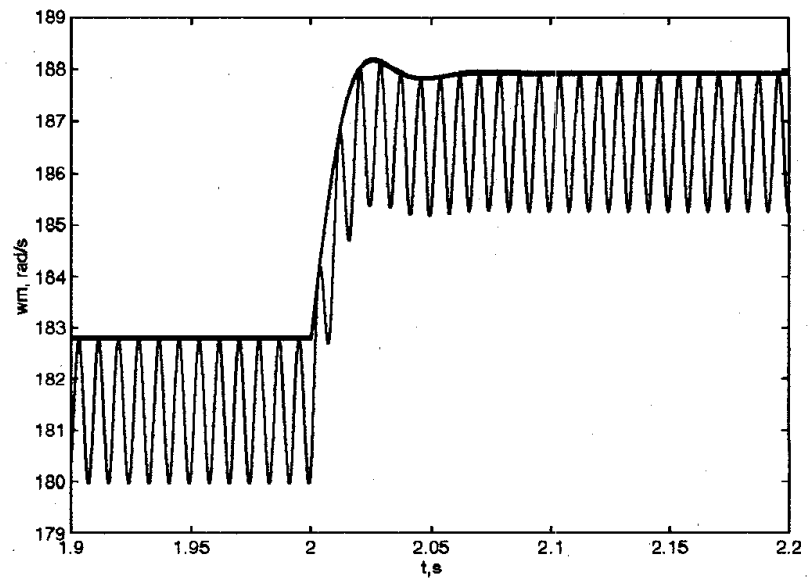

Figure 1: Mechanical speed from the standard model and speed envelope from the phasor-dynamic model ( $\mathrm{dc}$ and 2-nd harmonic).

outstanding agreement in all plots; the simplification of (6) obtained by setting $X_{4}^{2}=0$ yields very similar results (except that the ripple in speed and torque is not included). 


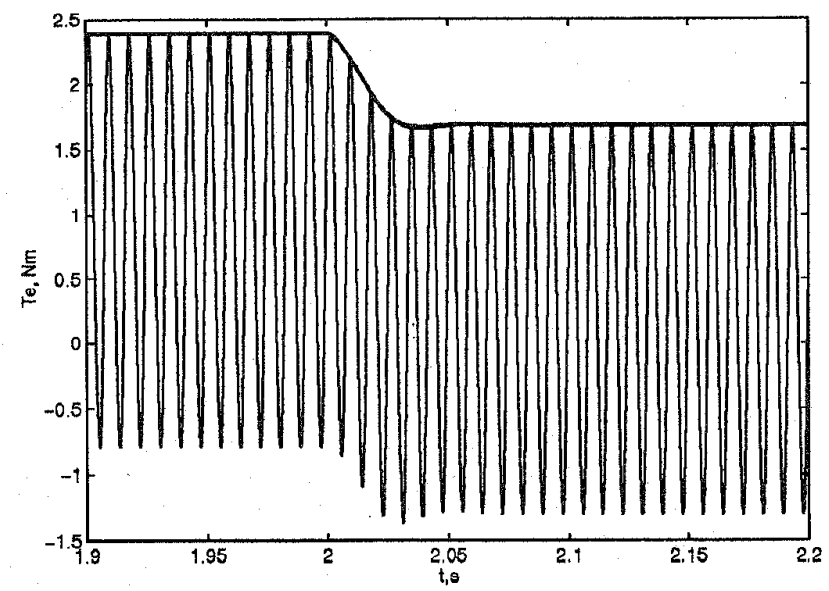

Figure 2: Instantaneous torque from the standard model and torque envelope from the phasor-dynamic model ( $\mathrm{dc}$ and 2-nd harmonic).

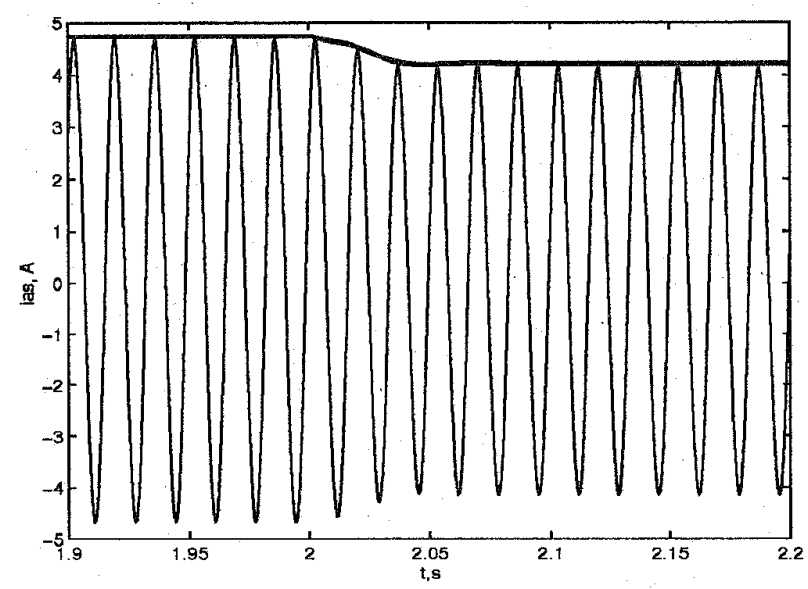

Figure 3: Stator current and its envelope.

\section{Small-Signal Models}

When model (6) is linearized around an operating point, it yields a 9 -th order model ( 7 -th if $\left\langle\omega_{r}\right\rangle_{2}$ is neglected). We list the eigenvalues (corresponding to the equilibrium point before the load step in the time-domain simulations) in Table 1 .

Observe that both 7 -th and 9-th order model have 3 pairs of eigenvalues that are similar. A participation factor analysis [14] shows that these eigenvalues are primarily influenced by the currents. This agrees with intuition that in an induction motor of this size the electrical subsystem should be considerably faster than the mechanical subsystem. The real eigenvalue at -86 practically corresponds to the dc component of the speed $\left\langle\omega_{r}>_{0}\right.$, while the "slow" complex pair in the 9-th order model corresponds to the second harmonic of the speed $\left.<\omega_{r}\right\rangle_{2}$.

The small-signal model corresponding to the lineariza-

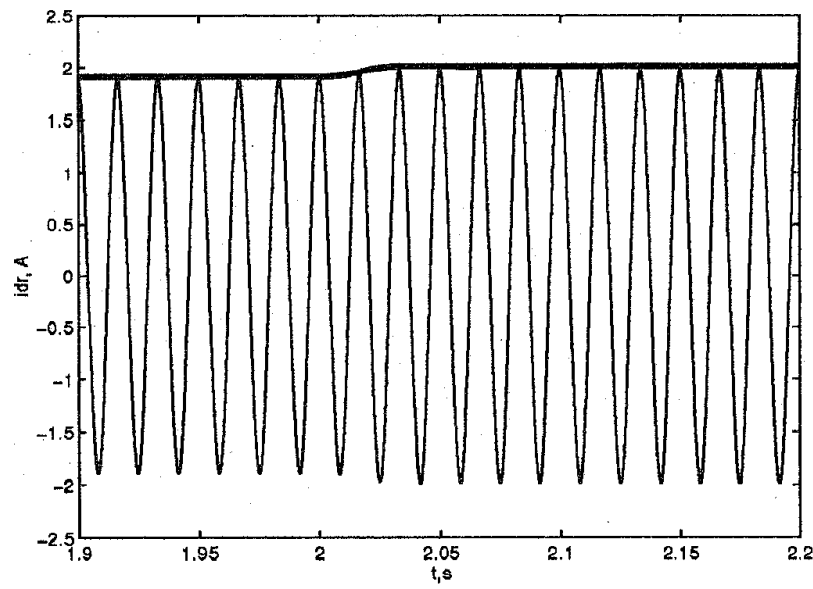

Figure 4: D-axis rotor current and its envelope.

Table 1: Eigenvalues

\begin{tabular}{||r|r|}
\hline 7th order & 9th order \\
\hline-86.48 & -86.25 \\
\hline$-75.57 \pm \mathrm{j} 130.44$ & $-75.11 \pm \mathrm{j} 131.80$ \\
\hline$-125.54 \pm \mathrm{j} 633.18$ & $-82.34 \pm \mathrm{j} 627.04$ \\
\hline$-259.27 \pm \mathrm{j} 390.65$ & $-267.23 \pm \mathrm{j} 377.48$ \\
\hline & $-35.82 \pm \mathrm{j} 772.00$ \\
\hline
\end{tabular}

tion of (a state-space version of) the time-domain model (4) around its steady state is periodic; due to the periodic input voltage. We have numerically evaluated its monodromy matrix $\Phi(T)$ (i.e, its state-transition matrix evaluated over one period $T$ ) [15]. For every eigenvalue $m$ of $\Phi(T)$ we have then calculated the corresponding eigenvalue $s$ of the statematrix that is obtained by a periodic ("Floquet") change of coordinates:

$$
s=\frac{1}{T} \ln m
$$

Note that since

$$
m=e^{s T}=e^{\left(s \pm j \ell \frac{2 \pi}{T}\right) T}
$$

for any integer $\ell$, there is actually a set of "Floquet" eigenvalues $s$ corresponding to each $m$. It can be shown [16] that in the case of linearizations of time-domain ("standard") models which are linear time-invariant (LTI), for each $\lambda$ of such model, the set $\lambda \pm j \ell 120 \pi$ corresponds to eigenvalues of the harmonic model (with $\mathrm{k}$ harmonics retained, $\ell \in[0,1, \ldots, k]$ ). If the same correspondence is to hold between the state matrix in Floquet coordinates and the harmonic model shown in Table 1 , from the numerically evaluated monodromy matrix $\Phi(T)$ we obtain the eigenvalues in Floquet coordinates as $(-85.40 ;-75.26 \pm j 132.23 ;-266.68)$. From this set, with additions of appropriate $j \ell 120 \pi$ factors, we have a good agreement with results presented in Table 1 for the harmonic model. 


\section{Equivalent Circuits}

Another use of model (6) is in deriving equivalent circuits for the single phase induction machine. To recover the commonly used circuit representations, we perform the following change of coordinates:

$$
\left[\begin{array}{l}
I_{s} \\
I_{+} \\
I_{-}
\end{array}\right]=\frac{1}{\sqrt{2}}\left[\begin{array}{ccc}
2 & 0 & 0 \\
0 & 2 & -2 j \\
0 & 2 & 2 j
\end{array}\right]\left[\begin{array}{c}
X_{1}^{1} \\
X_{2}^{1} \\
X_{3}^{1}
\end{array}\right]
$$

along with an identical change of coordinates for the voltage quantities. The $1 / \sqrt{2}$ premultiplying the transformation is simply to convert the quantities into rms values. The value 2 appearing in the transformation matrix takes into account the $X_{\ell}^{-k}=X_{\ell}^{k *}$ terms which are required to reconstruct the physical currents.

The resulting steady-state phasor equations for currents, with a little algebra, are

$$
\begin{aligned}
V= & r_{s} I_{s}+j X_{s} I_{s}+j \frac{X_{m}}{2}\left(I_{+}+I_{-}\right) \\
0= & \frac{r_{r} / 2}{\left(1-\frac{\left\langle\omega_{r}>_{0}\right.}{\omega_{b}}\right)} I_{+}+j \frac{X_{r}}{2} I_{+}+j \frac{X_{m}}{2} I_{s} \\
& +j \frac{X_{r}}{2} m_{+} I_{-}^{*}+j \frac{X_{m}}{2} m_{+} I_{s}^{*} \\
0= & \frac{r_{r} / 2}{\left(1+\frac{\zeta \omega_{r}>_{0}}{\omega_{b}}\right)} I_{-}+j \frac{X_{r}}{2} I_{-}+j \frac{X_{m}}{2} I_{s} \\
& +j \frac{X_{r}}{2} m_{-} I_{+}^{*}+j \frac{X_{m}}{2} m_{-} I_{s}^{*}
\end{aligned}
$$

where

$$
m_{+}=\frac{\frac{\left\langle\omega_{r}\right\rangle_{2}}{\omega_{b}}}{1-\frac{\left\langle\omega_{r}\right\rangle_{0}}{\omega_{b}}}
$$

and

$$
m_{-}=\frac{\frac{-\left\langle\omega_{r}\right\rangle_{2}}{\omega_{b}}}{1+\frac{\left\langle\omega_{r}\right\rangle_{0}}{\omega_{b}}} .
$$

This steady-state model has the circuit representation shown in Fig. 5 (with $X_{r}=X_{m}+X_{l r}, X_{s}=X_{m}+X_{l s}$ ). If a decision is made to neglect the second harmonic in the speed, then model (8) simplifies (as $m_{+}=m_{-}=0$ ), and the standard equivalent circuit for the single phase induction machine is recovered. The schematic presented in Fig. 6 is this simplified steady-state circuit representation. In the traditional interpretation, the $I_{+}$and $I_{-}$currents are associated with the forward and reverse rotating magnetic fields.

While the two equivalent circuits are different, in our example the corresponding steady-state currents and speed differ little, as shown in Table 2, where the discrepancies are in most cases $\leq 1 \%$. We also calculated the efficiency of the motor using the two equivalent circuits and compared the results to a value calculated from the simulation results. From the

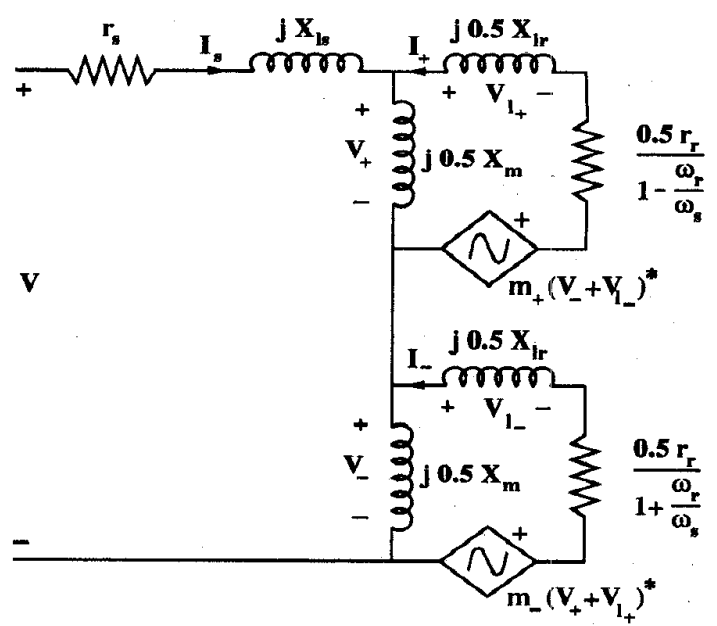

Figure 5: Complete equivalent circuit of a single-phase induction motor in steady-state

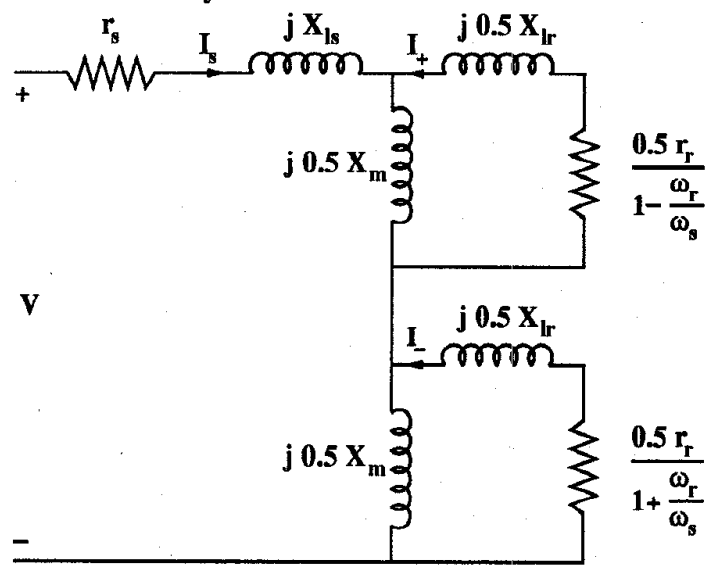

Figure 6: Simplified equivalent circuit of a single-phase induction motor in steady-state

time-domain simulations a value of $74.313 \%$ was calculated. Using the harmonic model the efficiency was calculated to be $74.317 \%$, while the simplified model yields $74.410 \%$. The improvement $(\approx 0.1 \%)$ could be of interest in motor design, where efficiency is the main figure of merit.

While the simplified circuit is well known, we point out that our derivation results in a more accurate model, and it makes no assumptions about the superposition of forwardand backward-rotating fields.

\section{CONCLUSIONS}

In this paper we have applied the generalized averaging technique to dynamical and stead-state modeling of a single-phase induction machine. We have developed a phasor-dynamic model of the machine that predicts its dynamics quite well, and can be used in high-performance control algorithms. It 
Table 2: Steady-state currents and speed, $T_{L}=0.8 \mathrm{Nm}$.

\begin{tabular}{|c|c|}
\hline \multicolumn{2}{|c|}{ 7th order } \\
\hline$i_{q s}(\mathrm{~A})$ & $1.253376-\mathrm{j} 1.991205$ \\
\hline$i_{q r}(\mathrm{~A})$ & $-1.245499+j 0.946732$ \\
\hline$i_{d r}(\mathrm{~A})$ & $0.944386+\mathrm{j} 0.026982$ \\
\hline$\left\langle T_{e}>_{0}(\mathrm{Nm})\right.$ & 0.8 \\
\hline$<\omega_{r}>_{0}(\mathrm{rad} / \mathrm{s})$ & 362.729540 \\
\hline$I_{+}(\mathrm{A})$ & $-2.437032+j 0.004693$ \\
\hline$I_{-}(\mathrm{A})$ & $-2.544962+\mathrm{j} 3.782237$ \\
\hline \multicolumn{2}{|c|}{ 9th order } \\
\hline$i_{q s}(\mathrm{~A})$ & $1.255087-\mathrm{j} 1.996277$ \\
\hline$i_{q r}(\mathrm{~A})$ & $-1.247127+\mathrm{j} 0.952068$ \\
\hline$i_{d r}(\mathrm{~A})$ & $0.946873+\mathrm{j} 0.029287$ \\
\hline$<\omega_{r}>_{0}(\mathrm{rad} / \mathrm{s})$ & 362.755375 \\
\hline$\left.<\omega_{r}\right\rangle_{2}(\mathrm{rad} / \mathrm{s})$ & $-1.160285-\mathrm{j} 0.780553$ \\
\hline$<T_{e}>_{0}(\mathrm{Nm})$ & 0.8 \\
\hline$\left\langle T_{e}\right\rangle_{2}(\mathrm{Nm})$ & $0.441393-\mathrm{j} 0.656126$ \\
\hline$I_{+}(\mathrm{A})$ & $-2.435682+\mathrm{j} 0.010390$ \\
\hline$I_{-}(\mathrm{A})$ & $-2.552828+\mathrm{j} 3.797881$ \\
\hline
\end{tabular}

is also well suited for numerical simulations, as it offers significant reductions in the required computational effort when compared with the standard model. In the steady-state the phasor-dynamic model reduces to an equivalent circuit which is a refinement of the conventional model, and leads to a more accurate efficiency calculation. A didactic advantage of the proposed approach is that it avoids superposition-type assumptions that are required in traditional modeling. The modeling framework described in this paper is open to further developments, as more harmonic components can be included in the analysis. This is particularly useful in studies of harmonics introduced by the mechanical subsystem, for example in the case of position-dependent loads.

\section{ACKNOWLEDGMENTS}

AMS and BCL are indebted to the National Science Foundation for support under Grants ECS-9410354 and ECS-9502636 (AMS) and ECS9409676 (BCL); AMS also thanks the Office of Naval Research for support under Grants N14-95-1-0723 and N14-97-1-0704.

\section{REFERENCES}

[1] A.E. Fitzgerald, C. Kingsley, Jr., and S. D. Umans, Electric Machinery, Fourth Edition, McGraw-Hill Book Co., New York, 1983.

[2] L.W. Matsch, Electromagnetic and Electromechanical Machines, IEP, New York, 1977.

[3] V. Del Toro, Electric Machines and Power Systems, Prentice-Hall, Englewood Cliffs, NJ, 1985.

[4] P.C. Sen, Principles of Electric Machinery and Power Electronics, John Wiley and Sons, New York, 1997.

[5] G.R. Slemon and A. Straughen, Electric Machines, AddisonWesley, Reading, MA, 1980.
[6] S.J. Chapman, Electric Machinery Fundamentals, Second Edition, McGraw-Hill, Inc., New York, 1991.

[7] D.V. Richardson and A.J. Caisse, Jr., Rotating Electric Machinery and Transformer Technology, Fourth Edition, Prentice-Hall, Upper Saddle River, NJ, 1997.

[8] T. Waldi, Electric Machines, Drives, and Power Systems, Third Edition, Prentice-Hall, Upper Saddle River, NJ, 1997.

[9] A.F. Puchstein and T.C Lloyd, Alternate Current Machines, John Wiley and Sons, New York, 1942.

[10] S.R. Sanders, J.M. Noworolski, X.Z. Liu and G.C. Verghese, "Generalized Averaging Method for Power Conversion Circuits," IEEE Transactions on Power Electronics, Vol. 6, No. 2, April 1991, pp. 251-259.

[11] S.R. Sanders, "On Limit Cycles and the Describing Function Method in Periodically Switched Circuits", IEEE Transactions on Circuits and Systems - Fundamental Theory and Applications, Vol. 40, No. 9, September 1993, pp. 564-572.

[12] P. Mattavelli, G.C. Verghese and A.M. Stankovic, "Phasor Dynamics in Thyristor-Controlled Series Capacitor Systems" IEEE Transactions on Power Systems, Vol. 12, No.3, August 1997, pp. 1259-1267.

[13] P. Krause, O. Wasynczuk and S. Sudhoff, Analysis of Electric $M a-$ chinery, IEEE Press, 1995.

[14] I.J. Perez-Arriaga, G.C. Verghese and F.C. Schweppe, "Selective Modal Analysis I: Heuristic Introduction," IEEE Transactions on Power Apparatus and Systems, Vol. 101, No. 9, September 1982, pp. 3117-3125.

[15] F. Bhathena, G.C. Verghese and M. Poloujadoff, "Stability Analysis of a non-Park-transformable electrical machine model," Mathematics and Computers in Simulation, Vol. 38, 1995, pp. 453-463.

[16] A.M.Stankovic, B.C. Lesieutre and T. Aydin, "Applications of Generalized Averaging to Synchronous and Induction Machines", 28-th North American Power Symposium, M.I.T., November 1996, pp. $277-282$.

\section{BIOGRAPHIES}

Aleksandar M. Stankovic obtained the Dipl. Ing. degree from the University of Belgrade, Yugoslavia in 1982, the M.S. degree from the same institution in 1986, and the Ph.D. degree from Massachusetts Institute of Technology in 1993, all in electrical engineering. He has been an Assistant Professor with the Department of Electrical and Computer Engineering at Northeastern University, Boston since 1993. Dr. Stanković is a member of IEEE Power Engineering, Power Electronics, Control Systems and Industry Applications Societies.

Bernard C. Lesieutre received his B.S., M.S. and Ph.D. degrees in electrical engineering from the University of Mlinois at UrbanaChampaign in 1986, 1988 and 1993, respectively. He is presently an Assistant Professor of Electrical Engineering at the Massachusetts Institute of Technology. His research interests include machine modeling and power system dynamics, stability and control. He is a member of Eta Kappa Nu.

Timur Aydin received the B.S. degree (1990) and the M.S. degree (1993) from Gazi University, Turkey, both in electrical engineering. He has been a Fh.D. student at Northeastern University since 1994. His research interests include modeling and simulation of electrical machines. 